\title{
A Web-Based Public Health Intervention to Reduce Functional Impairment and Depressive Symptoms in Adults With Type 2 Diabetes (The SpringboarD Trial): Randomized Controlled Trial Protocol
}

Judy Proudfoot ${ }^{12^{*}}$, PhD; Janine Clarke ${ }^{1^{*}}$, MPsychol (Clin), PhD; Jane Gunn ${ }^{3^{*}}, \mathrm{MD}, \mathrm{PhD}$; Susan Fletcher ${ }^{3^{*}}, \mathrm{PhD}$; Samineh Sanatkar ${ }^{1}$, PhD; Kay Wilhelm² ${ }^{2}$ MBBS, MD, FRANZCP; Lesley Campbell ${ }^{4}$, MBBS, FRCP (UK), FRACP AM; Nicholas Zwar ${ }^{5}$, MBBS, PhD; Mark Harris ${ }^{6}$, MBBS, PhD; Helen Lapsley², PhD; Dusan Hadzi-Pavlovic ${ }^{2}$, BSc, $^{2}$ MPsychol; Helen Christensen ${ }^{1,2}, \mathrm{PhD}$

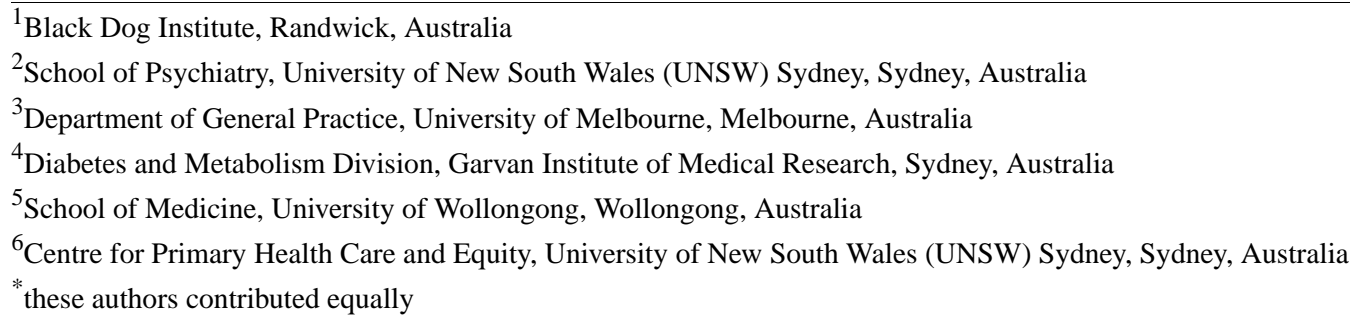

\section{Corresponding Author:}

Janine Clarke, MPsychol (Clin), PhD

Black Dog Institute

Hospital Road

Randwick, 2031

Australia

Phone: 61293823767 ext 23767

Fax: 61293828208

Email: janine.clarke@unsw.edu.au

\section{Abstract}

Background: Depressive symptoms are common in people with type 2 diabetes and contribute to adverse health consequences that substantially impact social and vocational function. Despite the existence of effective depression treatments, the majority of people with type 2 diabetes do not access these when needed. Web-based alternatives to more traditional psychotherapies offer a potential solution to reducing the personal and economic burdens of type 2 diabetes.

Objective: This paper outlines the protocol for a randomized controlled trial (RCT) of myCompass, a Web-based public health psychotherapy intervention, in people with type 2 diabetes. Fully automated, interactive, and delivered via the Internet without clinician support, myCompass teaches cognitive behavioral therapy-based skills and supports symptom monitoring to improve daily functioning and reduce mild-to-moderate mental health symptoms.

Methods: A two-arm RCT will be conducted. People with type 2 diabetes and mild-to-moderately severe depressive symptoms will be recruited from the community and general practice settings. Screening and enrollment is via an open-access website. Participants will be randomized to use either myCompass or an active placebo program for 8 weeks, followed by a 4-week tailing-off period. The placebo program is matched to myCompass on mode of delivery, interactivity, and duration. Outcomes will be assessed at baseline and at 3-month, 6-month, and 12-month follow-up. The primary study outcome is work and social functioning. Secondary study outcomes include depressive and anxious symptoms, diabetes-related distress, self-care behaviors, and glycemic control.

Results: Nationwide recruitment is currently underway with the aim of recruiting 600 people with type 2 diabetes. Recruitment will continue until October 2017.

Conclusions: This is the first known trial of a Web-based psychotherapy program that is not diabetes specific for improving social and vocational function in people with type 2 diabetes and mild-to-moderately severe depressive symptoms. With the 
increasing prevalence of type 2 diabetes and depression, a potentially scalable public health intervention could play a very large role in reducing unmet mental health need and ameliorating the personal and societal impact of illness comorbidity.

Trial Registration: Australian New Zealand Clinical Trials Registry (ANZCTR) Number: ACTRN12615000931572; https://www.anzctr.org.au/Trial/Registration/TrialReview.aspx?id=368109 (Archived by WebCite at http://www.webcitation.org/ 6rh3imVMh)

(JMIR Res Protoc 2017;6(8):e145) doi: 10.2196/resprot.7348

\section{KEYWORDS}

type 2 diabetes; depression; Web-based intervention

\section{Introduction}

\section{Background}

Type 2 diabetes is a common chronic and disabling disease and a major contributor to global disease burden [1]. Depression is frequently comorbid with type 2 diabetes and contributes independently to a range of adverse health outcomes that substantially compromise social and vocational functioning. These include greater diabetes symptom burden, poorer self-care, and increased risk of micro- and macrovascular complications and mortality [2]. In addition, the economic impact of disease comorbidity is considerable with functional disability, functional dependence, workplace productivity losses, health service use, and health care costs higher for people with both conditions than those with diabetes alone [2,3].

Research supports a bidirectional relationship between type 2 diabetes and depression [4]. Findings supporting increased risk of type 2 diabetes in people with depression [5] are generally explained with reference to physiological (eg, hypothalamicpituitary-adrenal axis dysfunction [6]), motivational (eg, poorer self-care, adiposity, and inactivity [6,7]), and/or pharmacologic (eg, impact of antidepressant medication on glycemic control [5]) factors. At the same time, increased risk of depression in people with type 2 diabetes is generally attributed to the emotional burden of living with a complex and demanding disease that often intrudes into normal lifestyle [8]. The likelihood of a reciprocal relationship between type 2 diabetes and depression makes the personal and societal impact of illness comorbidity potentially immeasurable [4].

Despite the existence of evidence-based therapies for depression in diabetes, including cognitive behavioral therapy (CBT) and antidepressant medication, upward of $60 \%$ of people with comorbid conditions do not receive mental health treatment [9]. In the primary health care setting, where most people with type 2 diabetes access medical treatment, low screening rates mean that depressive symptoms are often missed [9] and only a minority of patients who screen positive accept a referral for face-to-face support [10]. At the same time, personal, social, and structural barriers to seeking help-including privacy concerns and stigma, lack of support and poor relationships with health care providers, time and lifestyle constraints, financial cost, and lack of service availability-compromise access to satisfactory mental health care for many patients $[11,12]$. There is considerable opportunity, therefore, to reduce the personal and societal burden of illness by facilitating greater access to more flexible and cost-efficient mental health treatments for this patient group.

Internet delivery of evidence-based psychological therapies is a popular, clinically effective, and cost-effective means of increasing treatment reach; a small number of Web-based diabetes-specific interventions that directly target depression have been evaluated [13]. These include van Bastelaar et al's [14] adaptation of Lewinsohn's Coping with Depression Program and Nobis et al's [15] application of systematic behavioral activation and problem-solving therapy (ie, GesundheitsTraining.Online Mood Enhancer Diabetes [GET.ON MED]). Findings from these studies establish the symptom benefits of Internet-delivered self-help for diabetes patients with clinically relevant levels of depressive symptomatology. However, subthreshold depression is more prevalent in type 2 diabetes than clinical depression and is associated with increased functional limitation and disability, including reduced social and vocational performance $[16,17]$. As such, the effectiveness of electronically delivered psychotherapy as a treatment approach for type 2 patients with mild-to-moderate depressive symptoms also warrants rigorous scientific attention.

Proudfoot et al [18] have previously published controlled data showing that mild-to-moderate mental health symptomsincluding symptoms of depression, anxiety, and stress-are reduced following use of the broadly available Web-based program, myCompass, with treatment benefits extending to work and social functioning. More recently, in an uncontrolled pilot study, myCompass showed promise as an acceptable and effective treatment for depression and functional disability in people with type 1 and type 2 diabetes [19]. myCompass differs from the interventions described in van Bastelaar et al [14] and Nobis et al [15] in that it is a fully automated (ie, no therapist input) public health program that is generic in its therapeutic content (ie, not diabetes sensitive). An intervention approach that is capable of treating depressive symptoms without disease-specific modification is potentially a more efficient and accessible alternative to meeting the unmet mental health needs of people with type 2 diabetes. Generic skills may also assist the increasing number of individuals experiencing multi-morbidity, for whom depression co-occurs with somatic symptoms of multiple illnesses (eg, diabetes, heart disease, hypertension, and kidney disease) [20,21]. From a primary care perspective, a public health program may have important pragmatic advantages in the primary care setting, where time pressures often impede dissemination of, and prohibit practitioner training in, multiple disease-specific tools, and 
where treatment of multi-morbidity and undifferentiated physical and mental health symptoms is particularly relevant.

\section{Objectives}

This paper was prepared using the Standard Protocol Items: Recommendations for Interventional Trials (SPIRIT) guidance for presenting clinical trial protocols [22] and the Consolidated Standards of Reporting Trials of Electronic and Mobile HEalth Applications and onLine TeleHealth (CONSORT-EHEALTH) checklist [23]. The paper describes a randomized controlled trial (RCT) of the Web-based intervention, myCompass, in patients with comorbid type 2 diabetes and mild-to-moderately severe depressive symptoms. The primary aim of this RCT, called SpringboarD, is to determine whether treatment with myCompass improves work and social function for people with type 2 diabetes and mild-to-moderate depressive symptoms. As functional disability predicts further functional deterioration, functional dependence, and increased use of health services and health care costs [16], depression treatments that improve work and social functioning may substantially reduce the personal and economic burden of comorbid depression and type 2 diabetes. Specifically, we hypothesize that the intervention group will show significant improvement in self-reported functioning socially and in the workplace compared with a placebo-controlled condition.

Our secondary aim is to evaluate the impact of myCompass on a range of symptom- and disease-related variables known to impact a patient's disease management and blood glucose control. Specifically, in addition to depressive symptoms, we will examine whether myCompass is more effective than a placebo condition in improving anxiety symptoms and diabetes-related distress. Defined as a person's emotional adjustment to the chronic stressors specific to diabetes, diabetes-related distress is of particular interest due to its greater prevalence and potential role in mediating the relationship between depression and glycemic control [24]. While we have preliminary evidence suggesting that diabetes-related distress may improve following treatment with myCompass [19], replication of this finding in a controlled study is required.

\section{Trial Design}

The study is designed as a two-arm individually randomized RCT and is conducted entirely online. Participants allocated to the intervention group have full access to the myCompass program for 8 weeks, followed by a 4 -week tailing-off period to facilitate maintenance. Participants randomized to the control group have access to a placebo Internet-delivered program that is matched to myCompass on mode and duration of delivery and interactivity, but is void of therapeutic content. Participants in both groups have uninterrupted access to treatment as usual for their diabetes during the study.

\section{Methods}

\section{Participants, Interventions, and Outcomes}

\section{Study Setting}

The setting for this study is Australia. An estimated 1 million Australian adults $-5 \%$ of the population-had self-reported type 2 diabetes in September 2016, and rates are similar across metropolitan, regional, and remote areas [25].

\section{Eligibility Criteria}

This study focuses on adults with type 2 diabetes and mild-to-moderately severe depressive symptoms. People are eligible to take part if they are aged 18-75 years, screen positive for depression on the 2-item Patient Health Questionnaire (PHQ-2) (ie, score $\geq 2$ ) [26], and have access to an Internet-enabled device (eg, computer, tablet, and/or mobile phone). People who screen positive for depression complete the full 9-item Patient Health Questionnaire (PHQ-9) at screening so that the level of symptom severity can be determined.

Exclusion criteria include inability to read English with ease, severe depressive symptoms on the full PHQ-9 (ie, score >19), probable psychosis as measured by the psychosis screener developed for the Australian National Mental Health and Wellbeing Survey [27], currently receiving face-to-face counseling or therapy for depression, changed antidepressant medication in the previous 2 months, high suicide risk as assessed by item 9 of the PHQ-9, and previous use of the myCompass program.

\section{Interventions}

\section{Active Intervention}

The active intervention, myCompass [28], is a fully automated, self-help, public health intervention that is tailored to the user and has no therapist involvement in its delivery. Program tailoring occurs via users' responses to a symptom profiler completed at registration. In-built algorithms target the user's most salient symptoms and provide recommendations about the symptoms and/or behaviors they might consider monitoring and the treatment modules likely to be of greatest therapeutic benefit. There is flexibility, however, for users to choose their own set of self-monitoring dimensions and treatment modules (see Figures 1-4).

Self-monitoring of symptoms and lifestyle factors occurs in real time via mobile (eg, phone or tablet) and stationary (eg, desktop computer) devices. Users can self-monitor up to three symptoms and behaviors of their choice at any one time-selected from a list of 20 - or three that are recommended to them by the program. Each symptom is rated on a 10-point scale. At the time of rating, users also provide contextual information about where they are, what they are doing, and who they are with using a series of drop-down menus. Users can schedule short message service (SMS) text message or email reminders to facilitate self-monitoring - frequency of reminders is determined by the user; receive and print graphical feedback about their monitoring, including contextual information, on their phone or computer to monitor change and assist identification of triggers; and elect to receive helpful facts, mental health care tips, or motivational statements by SMS text message or email.

myCompass treatment modules were developed by mental health professionals at the Black Dog Institute in Sydney, Australia. The program contains 14 skill-building modules derived from CBT, interpersonal psychotherapy, problem-solving therapy, 
and positive psychology that are interactive and available for completion on computer and tablet devices. Module content covers topics such as Managing Fear and Anxiety, Tackling Unhelpful Thinking, Managing Loss and Major Life Change, and Solving Problems. Each module comprises three 10-minute sessions and includes home practice tasks for completion between the weekly sessions to promote skill generalization. The module targeting stress in diabetes has been deactivated for trial participants, to ensure that the intervention is a generic public health program. Participants are encouraged to complete at least three modules during the intervention period, during their own time.

User privacy is managed by a password-protected log-on and by ensuring that user-generated data (ie, self-monitoring ratings) are not stored on the user's phone, but are instead transferred via the Internet using Secure Sockets Layer protocol, which encrypts transmitted data by rendering it unreadable to anyone other than the intended recipient, and by storing the data in secure servers. The data is reidentifiable only with the list of study participant codes, to which only the named researchers will have access and which will be stored in a password-protected file separate from the study data. Registering to use myCompass is free and users are not billed for the SMS text messages they receive.

Participants randomized to myCompass have access to the full program for 8 weeks, followed by a 4-week tailing-off period during which only the symptom-monitoring function will be accessible. Research has shown that adherence with online interventions is improved when users receive program feedback that is personalized in its content [29]. For this reason, myCompass users receive automated personalized feedback via email about their use of the program's self-monitoring and module functions at fixed intervals (ie, weeks 1, 3, 5, and 7).

Figure 1. Screenshot of myCompass landing page.

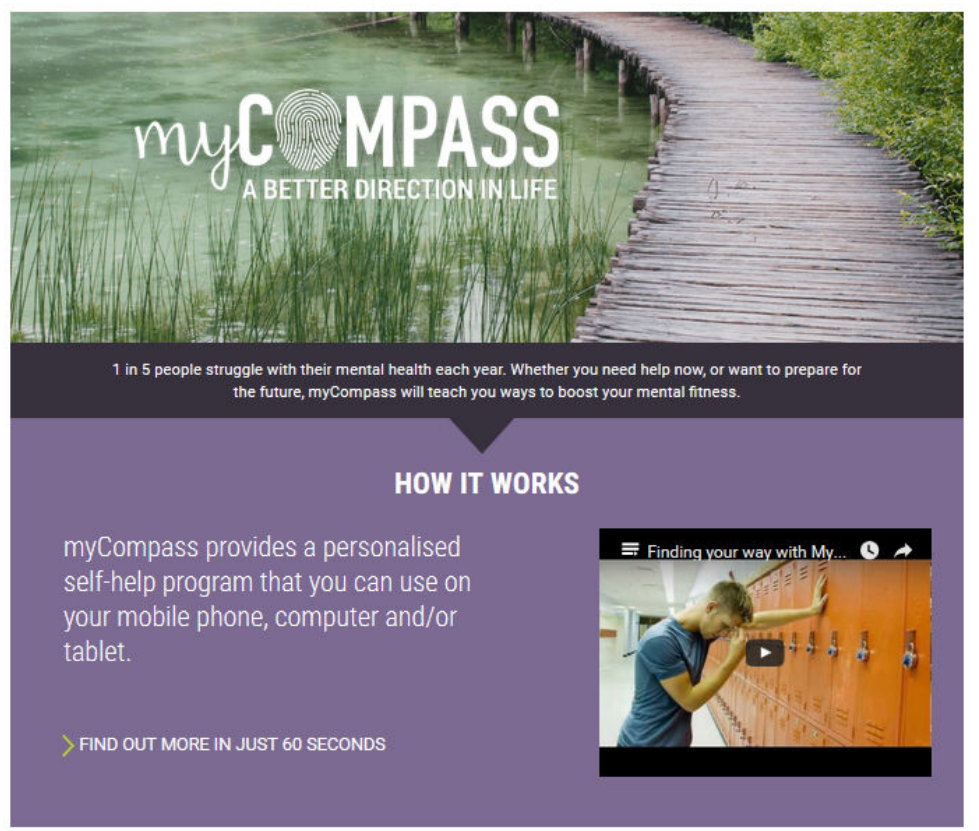

YOUR MENTAL HEALTH TOOLKIT

Learn new ways to deal with thoughts, feelings and behaviours that cause your trouble. You will have a tool-kit of strategies to use when you need them.

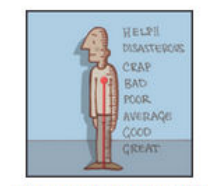

HOW ARE YOU TRAVEUING?

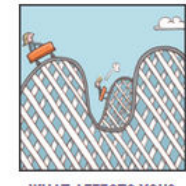

WHAT AFFECTS YOU?

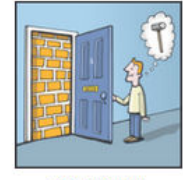

TAKE ACTION 
Figure 2. Screenshot of myCompass self-monitoring page.

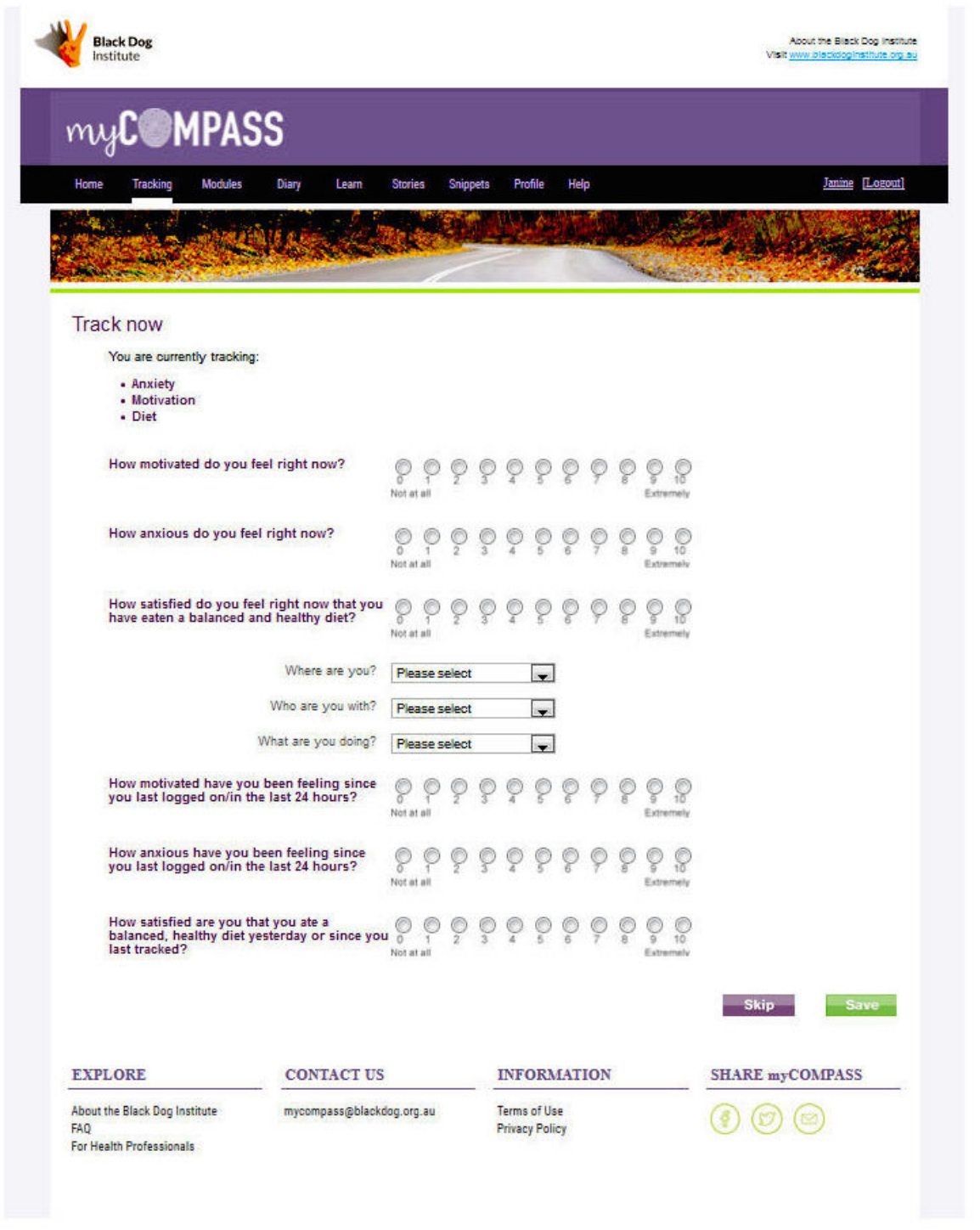


Figure 3. Screenshot of a page from the myCompass module Tackling Unhelpful Thinking.

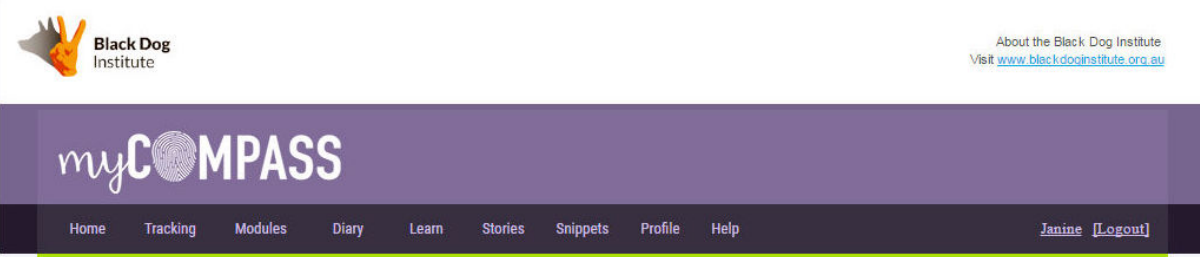

Tackling Unhelpful Thinking

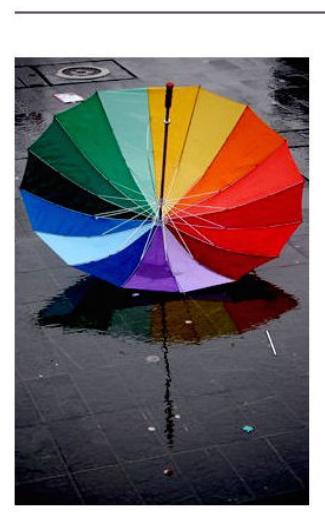

Thoughts and feelings

Thoughts are not the same as feelings. We feel 'feelings' - happiness, sadness, anger, and fear - in our bodies. For example, a spurt of energy, a sinking feeling, muschle tension or a racing heart. Thoughts are
different. We hold thoughts in our head - it's what we say to ourselves. Some people call their thoughts 'self-
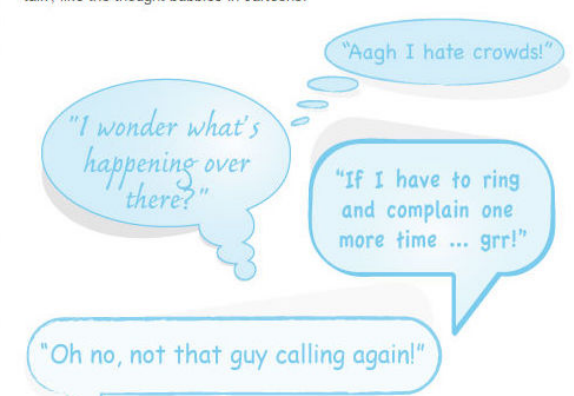

Of course, thoughts and feelings do affect each other. It is hard to know which comes first, and in some ways it doesn't really matter. But we do know that some thinking patterns can make us feel depressed. If $\mathrm{w}$ canes, we can change the wath, ce feet. It takes practice, but it is possible.

Figure 4. Screenshot of myCompass graphical feedback.

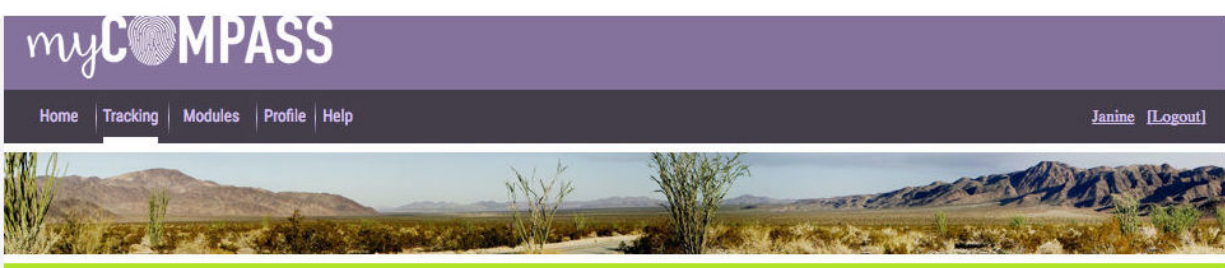

\section{Reports}

Reports are here to help you identify patterns in what you choose to track. By default we display the last 7 days data but you can customise them as you need.
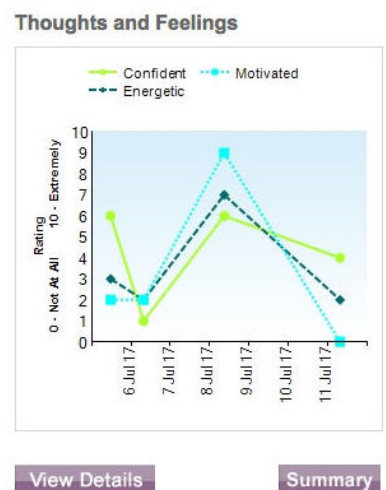


\section{Placebo Intervention}

The placebo program, Healthy Lifestyles, was adapted from a control program used in previous studies by members of the research team. Like myCompass, the program offers users program tailoring at the outset, followed by access to a range of interactive modules containing health- and lifestyle-related information, including skin care and eye health. It contains no therapeutic content, has high face validity as a health and lifestyle intervention, and has previously shown no symptom impact [30]. Participants have full access to the placebo condition for 8 weeks, plus a 4-week tailing-off period (see Figures 5-7).

To further replicate the interactivity of myCompass, users of the placebo program receive an email at weeks $1,3,5$, and 7 containing a brief reminder to log into the program, but no feedback about their use of the program. They also receive a weekly email containing a factual statement about a health or lifestyle issue.

Figure 5. Screenshot of landing page for placebo program, Healthy Lifestyles.

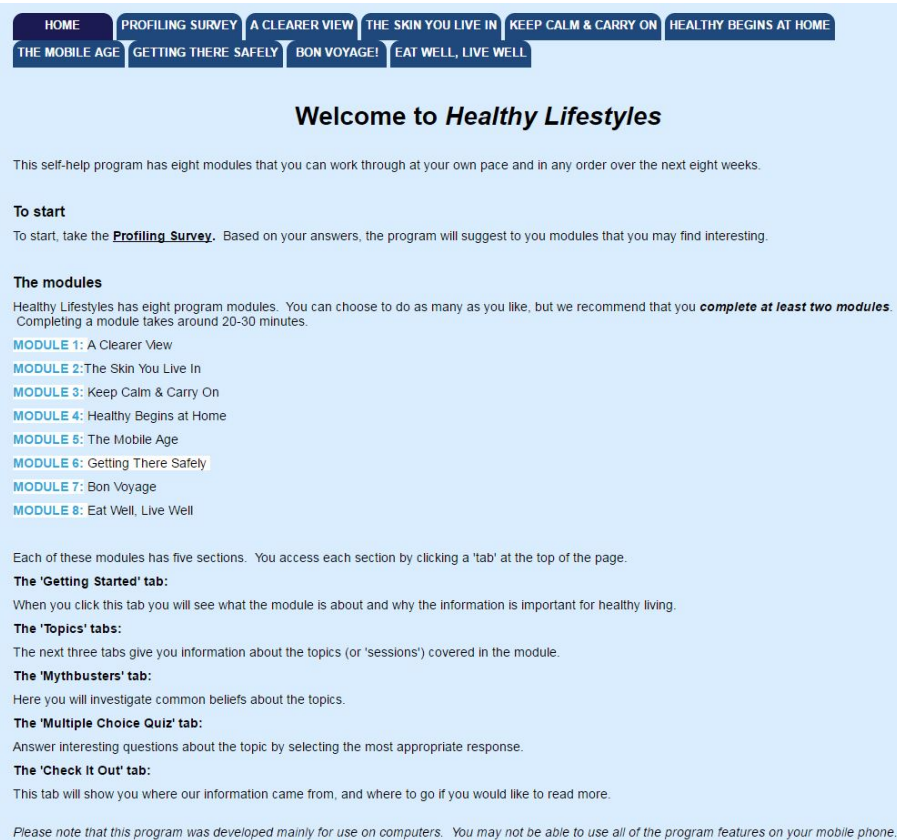

Figure 6. Page from the Healthy Lifestyles module, The Skin You Live In.

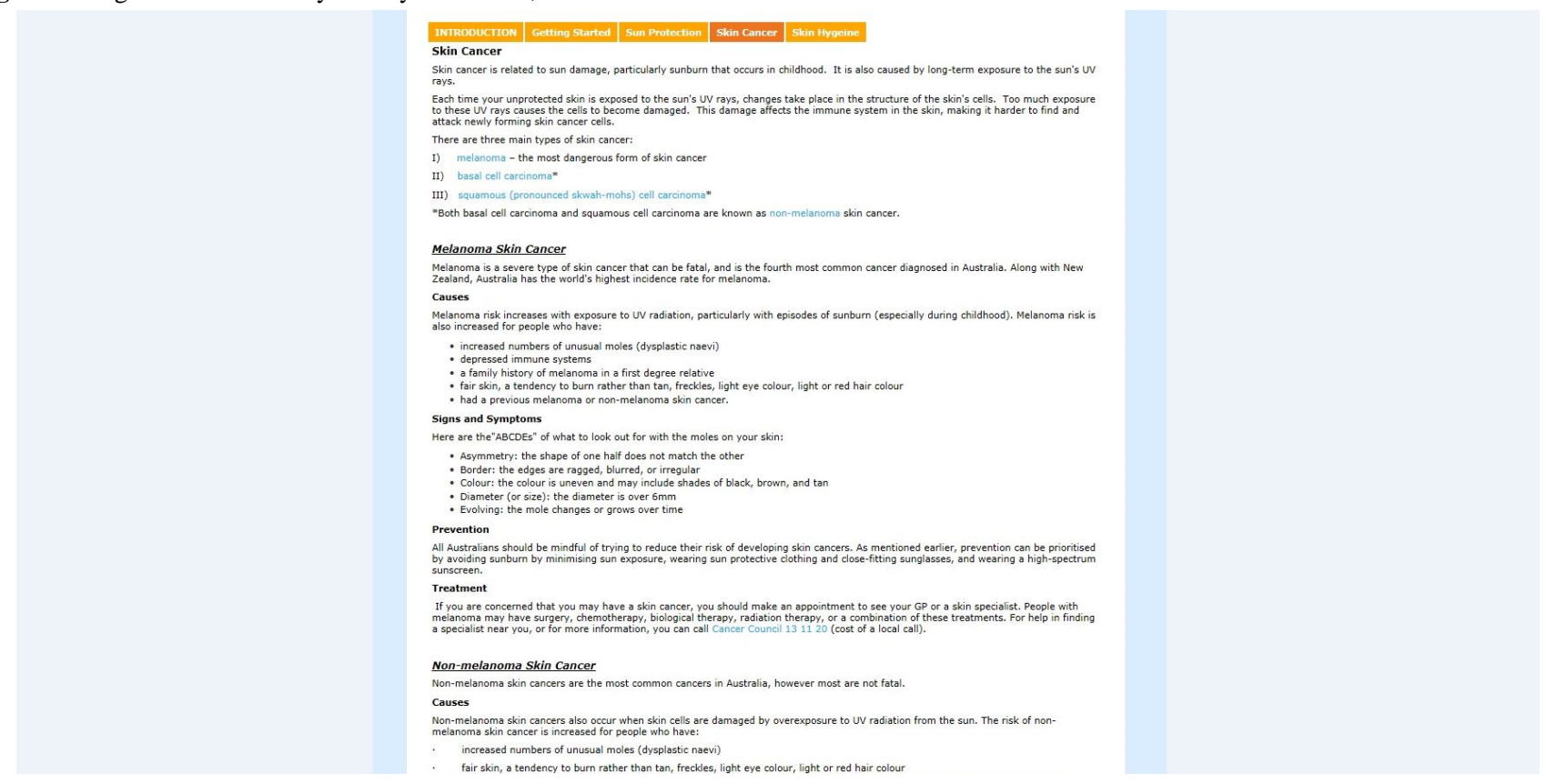


Figure 7. Sample interactive task from the Healthy Lifestyles module.

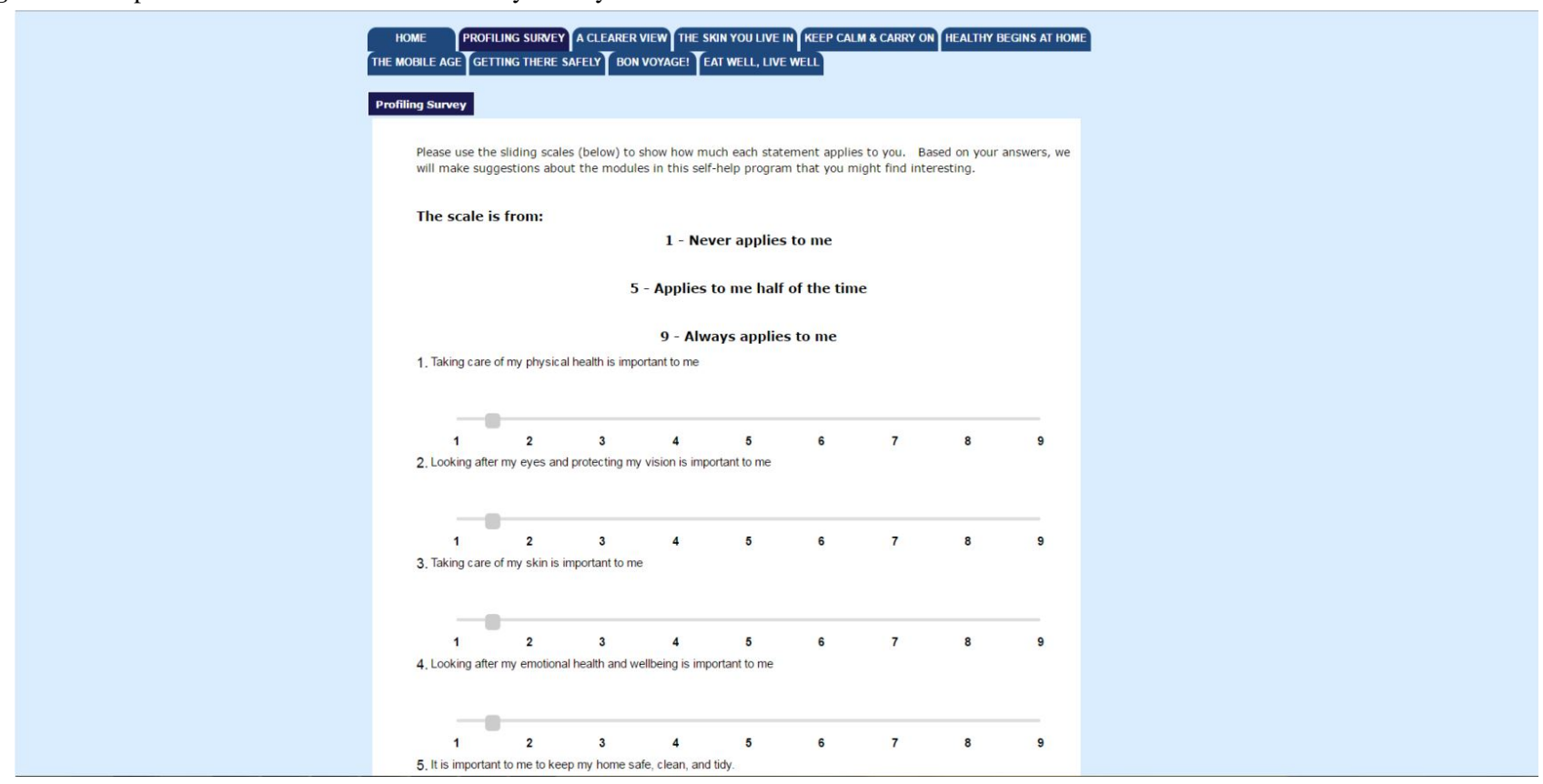

Table 1. Assessments completed at assessment phases.

\begin{tabular}{|c|c|c|c|c|c|}
\hline \multirow[t]{2}{*}{ Assessments } & & \multicolumn{4}{|c|}{ Assessment phase } \\
\hline & & $\mathrm{T} 1^{\mathrm{a}}$ & $\mathrm{T} 2^{\mathrm{b}}$ & $\mathrm{T} 3^{\mathrm{c}}$ & $\mathrm{T} 4^{\mathrm{d}}$ \\
\hline \multicolumn{6}{|l|}{ Baseline } \\
\hline & Demographic data & $\mathrm{X}$ & & & \\
\hline & Disease/treatment data & $\mathrm{X}$ & $\mathrm{X}$ & $\mathrm{X}$ & $\mathrm{X}$ \\
\hline & Mental health history & $\mathrm{X}$ & & & \\
\hline Primary outcome & Work and Social Adjustment Scale [31] & $\mathrm{X}$ & $\mathrm{X}$ & $\mathrm{X}$ & $\mathrm{X}$ \\
\hline \multicolumn{6}{|c|}{ Secondary outcomes } \\
\hline & Patient Health Questionnaire-9 [26] & $\mathrm{X}$ & $\mathrm{X}$ & $\mathrm{X}$ & $\mathrm{X}$ \\
\hline & Diabetes Distress Scale [32] & $\mathrm{X}$ & $\mathrm{X}$ & $\mathrm{X}$ & $\mathrm{X}$ \\
\hline & Generalized Anxiety Disorder Scale [33] & $\mathrm{X}$ & $\mathrm{X}$ & $X$ & $\mathrm{X}$ \\
\hline & Self-Management Profile for Type 2 Diabetes Scale (behavior items only) [34] & $\mathrm{X}$ & $\mathrm{X}$ & $\mathrm{X}$ & $\mathrm{X}$ \\
\hline & Glycosylated hemoglobin (HbA1c) & $\mathrm{X}$ & & $\mathrm{X}$ & $\mathrm{X}$ \\
\hline & Days out of role [35] & $\mathrm{X}$ & $\mathrm{X}$ & $\mathrm{X}$ & $\mathrm{X}$ \\
\hline & Health service utilization & $\mathrm{X}$ & $\mathrm{X}$ & $\mathrm{X}$ & $\mathrm{X}$ \\
\hline
\end{tabular}

${ }^{\mathrm{a}} \mathrm{T} 1$ : baseline assessment and allocation to intervention or placebo group.

${ }^{\mathrm{b}} \mathrm{T} 2$ : completion of intervention period and online postintervention assessment.

${ }^{\mathrm{c}} \mathrm{T} 3$ : completion of 6-month follow-up assessment.

${ }^{\mathrm{d}} \mathrm{T} 4$ : completion of 12-month follow-up assessment.

\section{Outcomes}

A summary of assessments completed online by participants at baseline, postintervention, and follow-up is presented in Table 1 [26,31-35].

The primary outcome is work and social functioning, which is measured by the Work and Social Adjustment Scale (WSAS) [31]. The WSAS is a psychometrically sound measure of the impact of mental health problems on daily functioning in five domains: work, social leisure activities, private leisure activities, home management, and personal relationships $[31,36]$. Scores range from 0 to 40 , with higher scores indicating poorer adjustment.

Secondary outcomes include the PHQ-9, the Diabetes Distress Scale [32], the 7-item Generalized Anxiety Scale [33], and the Self-Management Profile for Type 2 Diabetes Scale [34]. 
At baseline and again at 6- and 12-month follow-up, glycosylated hemoglobin $(\mathrm{HbA} 1 \mathrm{c})$ data will also be collected from participants' medical records, with their consent. The HbA1c test is a blood test showing a person's average blood glucose level over the previous 3 months and is measured as part of routine clinical care to monitor long-term blood sugar control in people with diabetes. In all cases, the most recent result will be obtained.

Additional measurements include collection of disease-related data (eg, age of onset and treatment regimen), sociodemographic data (eg, age, gender, education, and occupation), and mental health history data (eg, service use and previous diagnoses) at baseline. Service utilization and supports, including medication, received for problems related to mental health and diabetes are also assessed at each assessment point. Days out of role are measured using an item from the World Health Organization Disability Assessment Schedule that asks people to note the number of days in the previous 30 that they were completely unable to perform their work or normal activities because of problems with their physical or mental health [35].

At the conclusion of the trial, data indicating the extent of participant engagement with myCompass will be extracted from the program, including frequency of log-ins, number of modules started and completed, and self-monitoring frequency.

\section{Participant Timeline}

Participant consent, screening, and assessment takes place online via a secure study-specific website [37]. After providing informed consent, potential participants complete the online screening tool to determine eligibility. Unsuitable candidates receive automated feedback explaining the reason for their ineligibility; details of crisis supports and other self-help and face-to-face resources are provided to individuals, as appropriate.

Eligible candidates are registered in a secure Web-based platform where they complete the baseline questionnaire and are immediately allocated randomly to either the intervention program or control program for a period of 12 weeks.
Subsequent assessment points coincide for both groups at postintervention (12 weeks) as well as 6 and 12 months after randomization (see Figure 8).

\section{Sample Size}

The primary study outcome is work and social functioning, which is measured by the WSAS [31]. In a previous study comparing myCompass with an active control intervention, Proudfoot et al [18] obtained an average between-group effect size — based on estimated marginal means — of Cohen $d=0.3$ for work and social functioning. We calculated the sample size for this study using a series of $t$ tests (cross-sectional comparison of arms) with alpha $=.05$ and desired power $=80 \%$. The sample size required was $\mathrm{N}=350$ (175 per arm).

We cross-checked the sample size calculation using a reduction of 5 points on our secondary outcome, the PHQ-9, and got a similar outcome. A 5-point reduction on the PHQ-9 is considered an adequate treatment response [26].

Previous studies indicate attrition rates of approximately $40 \%$ in eHealth studies, generally [38]. As such, we aim to recruit 300 participants in each arm of the study (ie, 600 in total) for sufficient statistical power for completer analyses.

\section{Recruitment}

Study participants are being recruited via promotional materials distributed in general practice settings in New South Wales and Victoria-where the majority of Australians with type 2 diabetes reside - and disseminated nationally via print advertisements; social media posts, including Facebook and Twitter; clinical research registries; and other publicity channels of state and local diabetes stakeholder groups and the Black Dog Institute.

Promotional materials invite interested candidates to visit the SpringboarD Project website [37] to provide consent and complete the screening tool. The recruitment message for the study focuses on learning new ways to deal with stress and distress and live active and emotionally healthier lives with type 2 diabetes. Recruitment has commenced and will continue until October 2017 or until our sample target is reached. 
Figure 8. SpringboarD participant flow diagram.

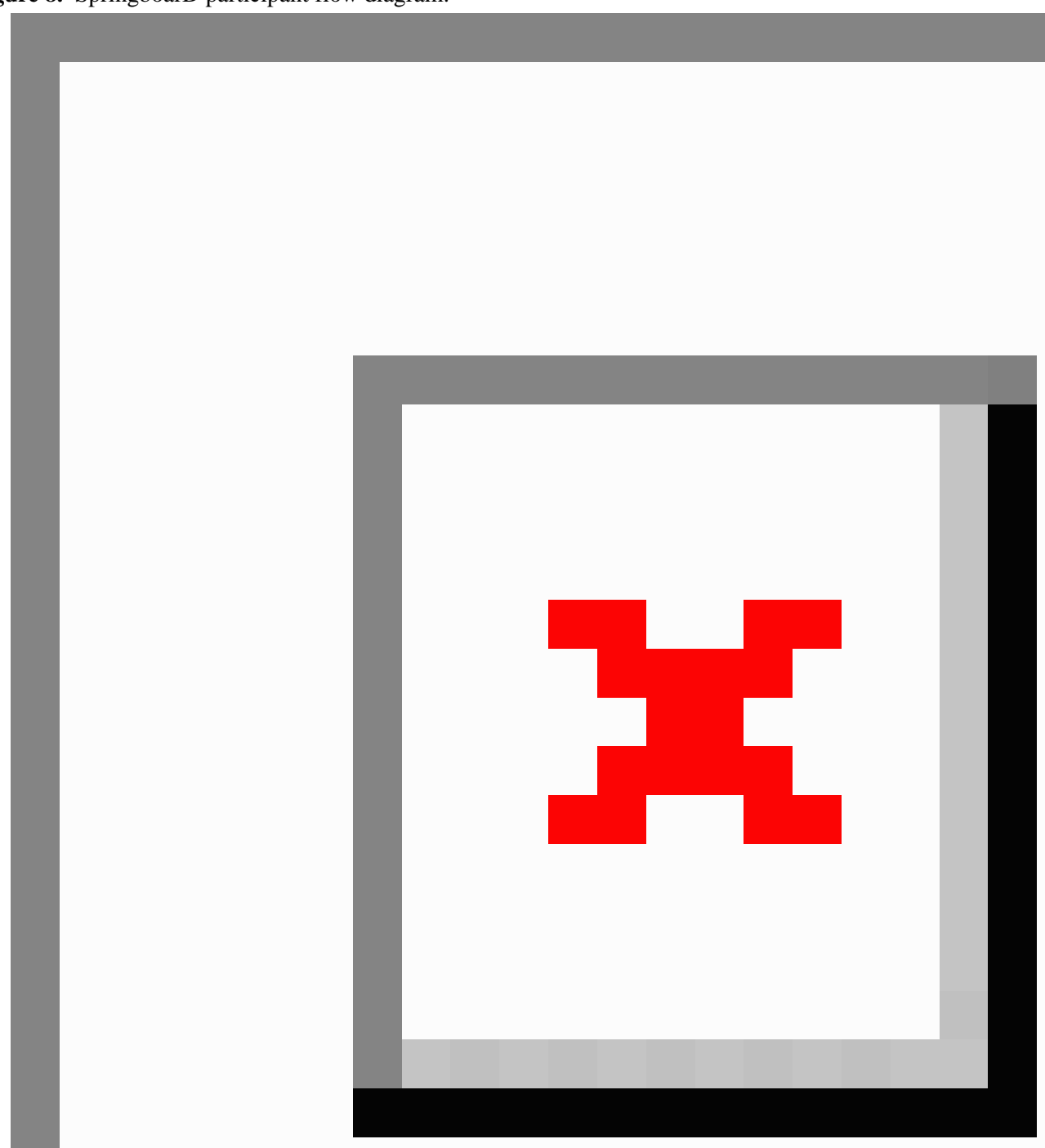

\section{Assignment of Interventions}

\section{Allocation}

We are using computerized blocked randomization with blocks of eight to assign participants to the two treatment conditions. Randomization to the intervention and placebo program occurs immediately after a participant completes the baseline measures using an automated system built into the study software. In this way, the allocation sequence is applied without the researchers' knowledge.

\section{Blinding}

The placebo program has been developed to replicate the mode of delivery, interactivity, and duration of myCompass and participants in each group will be treated equally by the research team. It is not possible, however, to assume that participants will remain blind to study allocation during the intervention and follow-up periods.

\section{Data Collection, Management, and Analysis}

\section{Data Collection}

The majority of primary and secondary outcome data is being collected electronically via standardized self-report questionnaires that are completed by logging into the SpringboarD study website (described above). At each assessment point, the website sends a unique link to the study questionnaire via email. Questionnaire data is maintained on a secure server at the University of New South Wales (UNSW) 
Sydney and is downloaded periodically for storage in a password-protected data file accessible by two project personnel (JC and SS). HbA1c data is collected from each participant's general practitioner (GP) via phone, mail, and/or facsimile.

\section{Retention}

Participant attrition is a major concern in studies of unguided interventions [38]. To facilitate trial retention, we are utilizing a combination of strategies covering various themes that have been used with success in previous studies. First, a systematic schedule of push messages, including personalized email and telephone prompts and reminders, is being implemented to motivate questionnaire completion at each of the postintervention and follow-up assessments [39]. Second, Aus $\$ 30$ gift vouchers are provided to participants who return completed questionnaires at each time point to compensate them for the time and effort they have expended [40]. Third, a sense of project identity is maintained by using a study logo and design template (ie, set fonts, formatting, and colors) to create the SpringboarD brand, and by distributing quarterly newsletters to participants to update them on trial progress [41]. Finally, a phone call from the research team within a week of registration provides participants a minimum level of human contact and the team an opportunity to express thanks, provide encouragement, and confirm personal details [42].

\section{Statistical Analysis}

Participants in the intervention and control groups will be compared at baseline using chi-square tests for categorical data and $t$ tests for continuous data to assess randomization success. Treatment effects on primary and secondary outcomes will be evaluated by intention-to-treat analysis using mixed-models repeated measures (MMRM). In MMRM, no participant is removed from the analysis because all available data are used to obtain parameter estimates. Effect size will be measured using Cohen $d$. For all outcome measures, within- and between-group differences will be standardized to Cohen $d$ using the pooled standard deviation of the observed scores obtained at baseline. We plan to analyze contrasts between intervention and control groups at postintervention and at 3-month, 6-month, and 12-month follow-up.

Supplementary analyses will use data for completers and will also investigate whether there are any differences by recruitment source, duration of diabetes, and presence of comorbid conditions.

\section{Monitoring}

The integrity of the trial, including data collection and monitoring, trial progress, adverse events, and compliance with UNSW Sydney Human Research Ethics Committee (HREC) reporting procedures, is overseen by the Steering Committee consisting of the chief (JP) and associate investigators. The Steering Committee will meet biannually over the lifespan of the project. Adverse events may include unfavorable changes to mental health or diabetes control and may be related or unrelated to the study. As the study does not impact routine diabetes care and is examining the effect of an evidence-based intervention for people with mild-to-moderately severe depression (ie, serious mental illness is an exclusion criteria), no serious adverse events are anticipated and no interim analyses are planned.

\section{Ethics and Dissemination}

\section{Research Ethics Approval}

The SpringboarD study protocol and materials have been approved by the HREC at UNSW Sydney and registered with the Australia and New Zealand Clinical Trials Register (ACTRN12615000931572). Annual reports and substantive amendments to this protocol will be submitted to the HREC for approval by the chief investigator. The study coordinator (JC) is responsible for communicating protocol changes to relevant stakeholders, including the Australian New Zealand Clinical Trials Registry.

\section{Consent or Assent}

Information about the study is provided on the SpringboarD project website; individuals can choose to read the information online or download a PDF to keep. Consent is obtained online in a two-stage process. First, individuals consent to the study by checking a box at the end of the study information page and progressing to the page containing the eligibility screen. Eligible individuals are then provided the option of registering an account with the study website (ie, username and password); those who opt not to register are also considered to have not consented to the trial.

Participants consent to the project team informing their treating GP of his or her involvement in the study to facilitate $\mathrm{HbA} 1 \mathrm{c}$ data collection; they also provide a point of emergency contact should a participant score in the severely distressed range of the PHQ-9 (ie, score >19) or be at risk of self-harm (ie, score 3 on item 9 of the PHQ-9) at any assessment point. GPs are informed by mail within 2 weeks of their patient's enrolment, at which time they are requested to inform the research team if a diagnosis of type 2 diabetes has not been given.

\section{Confidentiality}

The eligibility screen is conducted anonymously such that no personal information about potential participants is collected. Only eligible individuals provide identifying information that is downloaded and stored separately from study data in a password-protected file.

\section{Ancillary and Posttrial Care}

At the conclusion of the trial, the active intervention will be made available to all participants in the control group.

\section{Results}

Nationwide recruitment is currently underway with the aim of recruiting 600 people with type 2 diabetes. Recruitment will continue until October 2017.

\section{Discussion}

\section{Principal Findings}

Treatment of depressive symptoms in people with type 2 diabetes might help to improve short- and long-term social and vocational functioning. Internet-delivered psychotherapy is an 
effective treatment for depression in people with type 2 diabetes; however, few studies have focused on mild-to-moderately severe depressive symptoms where treatment need in diabetes patients is greatest. Rarely has social and vocational function been evaluated in studies of online depression treatments in type 2 diabetes; the effectiveness of a generic Internet-based program has not been studied in this patient group. This study will shed light on whether an Internet-delivered public health program has the potential to reduce unmet treatment need and lessen the personal and societal impact of mild-to-moderately severe depressive symptoms within the context of type 2 diabetes.

\section{Limitations}

Trials of self-guided interventions frequently report high rates of attrition, including study dropout (ie, questionnaire noncompletion) and/or disengagement from the program [38]. Study attrition introduces selection bias and potential misrepresentation of treatment effects. To reduce the impact of actual participant dropout, we will recruit a substantially larger sample than is required on the basis of our sample size calculation. To minimize potential study dropout, we will utilize a combination of strategies that have been shown to positively impact retention in previous trials, including email and telephone prompts and reminders [39], recompense for questionnaire completion [40], and activities aimed at keeping the study "front of mind" and engaging for older participants [41,43]. In addition, so that questionnaire completion is not contingent upon program use, participants will access the study questionnaire outside the intervention (ie, via a link sent to a nominated email account). To maximize program use, automated program reminders will be sent by email to participants at biweekly intervals [29].

Volunteer bias is another possible weakness of this study, as our recruitment techniques may yield a sample that is healthier [44] and more highly motivated to learn new skills and engage with self-guided therapy. Problems occur, for example, if participation in our study is reflective of better health and a broader personal commitment to self-improvement of diabetes outcomes and daily functioning, as this is likely to result in within-group changes that are larger than for a less motivated and more representative sample of type 2 diabetes patients. However, since myCompass is broadly available for self-referral and use whenever and wherever people choose, the recruitment processes are consistent with both the self-help nature of the intervention and its eventual use in the type 2 diabetes population.

\section{Conclusions}

The personal and economic costs of comorbid type 2 diabetes and depressive symptoms are substantial. However, the development of Internet-delivered interventions offers a potential solution to mitigate these impacts [13-15]. myCompass is a broadly available and efficacious public health intervention that can be delivered at minimal cost [45]. It therefore presents itself as a potentially effective and timely option for reducing unmet mental health need and ameliorating the personal and societal impact of co-occurring depression and type 2 diabetes.

\section{Acknowledgments}

The authors acknowledge support from the Australian Government's National Health and Medical Research Council (grant No. 1083116).

\section{Conflicts of Interest}

None declared.

\section{Multimedia Appendix 1 \\ CONSORT-EHEALTH checklist V1.6.1 [23].}

[PDF File (Adobe PDF File), 7MB-Multimedia Appendix 1]

\section{References}

1. Roy T, Lloyd CE. Epidemiology of depression and diabetes: A systematic review. J Affect Disord 2012 Oct;142 Suppl:S8-S21. [doi: 10.1016/S0165-0327(12)70004-6] [Medline: 23062861]

2. Katon WJ. The comorbidity of diabetes mellitus and depression. Am J Med 2008 Nov;121(11 Suppl 2):S8-S15 [FREE Full text] [doi: 10.1016/j.amjmed.2008.09.008] [Medline: 18954592]

3. Egede LE. Effects of depression on work loss and disability bed days in individuals with diabetes. Diabetes Care 2004 Jul;27(7):1751-1753. [Medline: 15220260]

4. Manigault KR. The bidirectional relationship between depression and diabetes. US Pharm 2016 Nov 17;41(11):26-29 [FREE Full text]

5. Semenkovich K, Brown ME, Svrakic DM, Lustman PJ. Depression in type 2 diabetes mellitus: Prevalence, impact, and treatment. Drugs 2015 Apr;75(6):577-587. [doi: 10.1007/s40265-015-0347-4] [Medline: 25851098]

6. Holt RI, de Groot M, Golden SH. Diabetes and depression. Curr Diab Rep 2014 Jun;14(6):491 [FREE Full text] [doi: 10.1007/s11892-014-0491-3] [Medline: 24743941]

7. Renn BN, Feliciano L, Segal DL. The bidirectional relationship of depression and diabetes: A systematic review. Clin Psychol Rev 2011 Dec;31(8):1239-1246. [doi: 10.1016/j.cpr.2011.08.001] [Medline: 21963669] 
8. Fisher EB, Chan JC, Nan H, Sartorius N, Oldenburg B. Co-occurrence of diabetes and depression: Conceptual considerations for an emerging global health challenge. J Affect Disord 2012 Oct;142 Suppl:S56-S66. [doi: 10.1016/S0165-0327(12)70009-5] [Medline: 23062858]

9. Hermanns N, Caputo S, Dzida G, Khunti K, Meneghini LF, Snoek F. Screening, evaluation and management of depression in people with diabetes in primary care. Prim Care Diabetes 2013 Apr;7(1):1-10 [FREE Full text] [doi: 10.1016/j.pcd.2012.11.002] [Medline: 23280258]

10. Fleer J, Tovote KA, Keers JC, Links TP, Sanderman R, Coyne JC, et al. Screening for depression and diabetes-related distress in a diabetes outpatient clinic. Diabet Med 2013 Jan;30(1):88-94. [doi: 10.1111/dme.12001] [Medline: 22924587]

11. Griffiths KM, Christensen H. Internet-based mental health programs: A powerful tool in the rural medical kit. Aust J Rural Health 2007 Apr;15(2):81-87. [doi: 10.1111/j.1440-1584.2007.00859.x] [Medline: 17441815]

12. Blixen CE, Kanuch S, Perzynski AT, Thomas C, Dawson NV, Sajatovic M. Barriers to self-management of serious mental illness and diabetes. Am J Health Behav 2016 Mar;40(2):194-204 [FREE Full text] [doi: 10.5993/AJHB.40.2.4] [Medline: 26931751]

13. Hadjiconstantinou M, Byrne J, Bodicoat DH, Robertson N, Eborall H, Khunti K, et al. Do Web-based interventions improve well-being in type 2 diabetes? A systematic review and meta-analysis. J Med Internet Res 2016 Oct 21;18(10):e270 [FREE Full text] [doi: 10.2196/jmir.5991] [Medline: 27769955]

14. van Bastelaar KM, Pouwer F, Cuijpers P, Riper H, Snoek FJ. Web-based depression treatment for type 1 and type 2 diabetic patients: A randomized, controlled trial. Diabetes Care 2011 Feb;34(2):320-325 [FREE Full text] [doi: 10.2337/dc10-1248] [Medline: 21216855]

15. Nobis S, Lehr D, Ebert DD, Berking M, Heber E, Baumeister H, et al. Efficacy and cost-effectiveness of a Web-based intervention with mobile phone support to treat depressive symptoms in adults with diabetes mellitus type 1 and type 2 : Design of a randomised controlled trial. BMC Psychiatry 2013 Nov 15;13:306 [FREE Full text] [doi:

10.1186/1471-244X-13-306] [Medline: 24238346]

16. Egede LE. Diabetes, major depression, and functional disability among U.S. adults. Diabetes Care 2004 Feb;27(2):421-428. [Medline: 14747223$]$

17. Schmitz N, Gariépy G, Smith KJ, Malla A, Boyer R, Strychar I, et al. Longitudinal relationships between depression and functioning in people with type 2 diabetes. Ann Behav Med 2014 Apr;47(2):172-179. [doi: 10.1007/s12160-013-9534-2] [Medline: 24046149]

18. Proudfoot J, Clarke J, Birch M, Whitton AE, Parker G, Manicavasagar V, et al. Impact of a mobile phone and Web program on symptom and functional outcomes for people with mild-to-moderate depression, anxiety and stress: A randomised controlled trial. BMC Psychiatry 2013 Nov 18;13:312 [FREE Full text] [doi: 10.1186/1471-244X-13-312] [Medline: 24237617]

19. Clarke J, Proudfoot J, Ma H. Mobile phone and Web-based cognitive behavior therapy for depressive symptoms and mental health comorbidities in people living with diabetes: Results of a feasibility study. JMIR Ment Health 2016 May 31;3(2):e23 [FREE Full text] [doi: 10.2196/mental.5131] [Medline: 27245948]

20. de Groot M, Anderson R, Freedland KE, Clouse RE, Lustman PJ. Association of depression and diabetes complications: A meta-analysis. Psychosom Med 2001;63(4):619-630. [Medline: 11485116 ]

21. Gunn JM, Ayton DR, Densley K, Pallant JF, Chondros P, Herrman HE, et al. The association between chronic illness, multimorbidity and depressive symptoms in an Australian primary care cohort. Soc Psychiatry Psychiatr Epidemiol 2012 Feb;47(2):175-184. [doi: 10.1007/s00127-010-0330-z] [Medline: 21184214]

22. Chan A, Tetzlaff JM, Gøtzsche PC, Altman DG, Mann H, Berlin JA, et al. SPIRIT 2013 explanation and elaboration: Guidance for protocols of clinical trials. BMJ 2013 Jan 08;346:e7586 [FREE Full text] [Medline: 23303884]

23. Eysenbach G, CONSORT-EHEALTH Group. CONSORT-EHEALTH: Improving and standardizing evaluation reports of Web-based and mobile health interventions. J Med Internet Res 2011 Dec 31;13(4):e126 [FREE Full text] [doi: 10.2196/jmir.1923] [Medline: 22209829]

24. Fisher L, Gonzalez JS, Polonsky WH. The confusing tale of depression and distress in patients with diabetes: A call for greater clarity and precision. Diabet Med 2014 Jul;31(7):764-772 [FREE Full text] [doi: 10.1111/dme.12428] [Medline: 24606397]

25. Type 2 Diabetes. Turner, Australia: National Diabetes Services Scheme (NDSS), Diabetes Australia; 2016 Sep 30. URL: https://static.diabetesaustralia.com.au/s/fileassets/diabetes-australia/6eb4569c-dabf-4187-8eab-a571eb3dc3f6.pdf [accessed 2017-01-17] [WebCite Cache ID 6nZnpdASe]

26. Kroenke K, Spitzer RL. The PHQ-9: A new depression diagnostic and severity measure. Psychiatr Ann 2002 Sep;32(9):509-515. [doi: 10.3928/0048-5713-20020901-06]

27. Degenhardt L, Hall W. The Association Between Psychosis and Problematic Drug Use Among Australian Adults: Findings From the National Survey of Mental Health and Well-being. Randwick, Australia: National Drug and Alcohol Research Centre, University of New South Wales; 2000. URL: https://ndarc.med.unsw.edu.au/sites/default/files/ndarc/resources/TR. 093.pdf [accessed 2017-07-04] [WebCite Cache ID 6riYMjFUO]

28. myCompass. URL: https://www.mycompass.org.au/ [accessed 2017-07-02] [WebCite Cache ID 6rfMEogzd] 
29. Clarke G, Eubanks D, Reid E, Kelleher C, O'Connor E, DeBar LL, et al. Overcoming Depression on the Internet (ODIN) (2): A randomized trial of a self-help depression skills program with reminders. J Med Internet Res 2005 Jun 21;7(2):e16 [FREE Full text] [doi: 10.2196/jmir.7.2.e16] [Medline: $\underline{\text { 15998607] }}$

30. Christensen H, Griffiths KM, Mackinnon AJ, Kalia K, Batterham PJ, Kenardy J, et al. Protocol for a randomised controlled trial investigating the effectiveness of an online eHealth application for the prevention of generalised anxiety disorder. BMC Psychiatry 2010 Mar 21;10:25 [FREE Full text] [doi: 10.1186/1471-244X-10-25] [Medline: 20302678]

31. Mundt JC, Marks IM, Shear MK, Greist JH. The Work and Social Adjustment Scale: A simple measure of impairment in functioning. Br J Psychiatry 2002 May;180:461-464 [FREE Full text] [Medline: 11983645]

32. Polonsky WH, Anderson BJ, Lohrer PA, Welch G, Jacobson AM, Aponte JE, et al. Assessment of diabetes-related distress. Diabetes Care 1995 Jun;18(6):754-760. [Medline: 7555499]

33. Spitzer RL, Kroenke K, Williams JB, Löwe B. A brief measure for assessing generalized anxiety disorder: The GAD-7. Arch Intern Med 2006 May 22;166(10):1092-1097. [doi: 10.1001/archinte.166.10.1092] [Medline: 16717171]

34. Peyrot M, Bushnell DM, Best JH, Martin ML, Cameron A, Patrick DL. Development and validation of the self-management profile for type 2 diabetes (SMP-T2D). Health Qual Life Outcomes 2012 Oct 05;10:125 [FREE Full text] [doi: 10.1186/1477-7525-10-125] [Medline: 23039868]

35. Üstün TB, Kostanjsek N, Chatterji S, Rehm J, editors. Measuring Health and Disability: Manual for WHO Disability Assessment Schedule (WHODAS 2.0). Geneva, Switzerland: World Health Organization; 2010.

36. Meyer B, Berger T, Caspar F, Beevers CG, Andersson G, Weiss M. Effectiveness of a novel integrative online treatment for depression (Deprexis): Randomized controlled trial. J Med Internet Res 2009 May 11;11(2):e15 [FREE Full text] [doi: 10.2196/jmir.1151] [Medline: 19632969]

37. SpringboarD. URL: https://springboard.blackdoghealth.org.au [accessed 2017-07-03] [WebCite Cache ID 6rfZH13S4]

38. Eysenbach G. The law of attrition. J Med Internet Res 2005 Mar 31;7(1):e11 [FREE Full text] [doi: 10.2196/jmir.7.1.e11] [Medline: 15829473]

39. Christensen H, Batterham P, Mackinnon A, Griffiths KM, Kalia HK, Kenardy J, et al. Prevention of generalized anxiety disorder using a Web intervention, iChill: Randomized controlled trial. J Med Internet Res 2014 Sep 02;16(9):e199 [FREE Full text] [doi: 10.2196/jmir.3507] [Medline: 25270886]

40. Brueton VC, Tierney J, Stenning S, Harding S, Meredith S, Nazareth I, et al. Strategies to improve retention in randomised trials. Cochrane Database Syst Rev 2013 Dec 03(12):MR000032 [FREE Full text] [doi: 10.1002/14651858.MR000032.pub2] [Medline: 24297482]

41. Robinson KA, Dinglas VD, Sukrithan V, Yalamanchilli R, Mendez-Tellez PA, Dennison-Himmelfarb C, et al. Updated systematic review identifies substantial number of retention strategies: Using more strategies retains more study participants. J Clin Epidemiol 2015 Dec;68(12):1481-1487 [FREE Full text] [doi: 10.1016/j.jclinepi.2015.04.013] [Medline: 26186981]

42. Andrews G, Williams A. Up-scaling clinician assisted Internet cognitive behavioural therapy (iCBT) for depression: A model for dissemination into primary care. Clin Psychol Rev 2015 Nov;41:40-48. [doi: 10.1016/j.cpr.2014.05.006] [Medline: 25043445]

43. Davies K, Kingston A, Robinson L, Hughes J, Hunt J, Barker S, et al. Improving retention of very old participants in longitudinal research: Experiences from the Newcastle 85+ study. PLoS One 2014;9(10):e108370 [FREE Full text] [doi: 10.1371/journal.pone.0108370] [Medline: 25302500]

44. Stoop CH, Nefs G, Pop VJ, Pouwer F. Screening for and subsequent participation in a trial for depression and anxiety in people with type 2 diabetes treated in primary care: Who do we reach? Prim Care Diabetes 2017 Jun;11(3):273-280. [doi: 10.1016/j.pcd.2017.02.006] [Medline: 28330680]

45. Solomon D, Proudfoot J, Clarke J, Christensen H. e-CBT (myCompass), antidepressant medication, and face-to-face psychological treatment for depression in Australia: A cost-effectiveness comparison. J Med Internet Res 2015 Nov 11;17(11):e255 [FRE Full text] [doi: 10.2196/jmir.4207] [Medline: 26561555]

\section{Abbreviations}

CBT: cognitive behavioral therapy

CONSORT-EHEALTH: Consolidated Standards of Reporting Trials of Electronic and Mobile HEalth Applications and onLine TeleHealth

GET.ON MED: GesundheitsTraining.Online Mood Enhancer Diabetes

GP: general practitioner

HbA1c: glycosylated hemoglobin

HREC: Human Research Ethics Committee

MMRM: mixed-models repeated measures

PHQ-2: 2-item Patient Health Questionnaire

PHQ-9: 9-item Patient Health Questionnaire

RCT: randomized controlled trial

SMS: short message service 
SPIRIT: Standard Protocol Items: Recommendations for Interventional Trials

UNSW: University of New South Wales

WSAS: Work and Social Adjustment Scale

Edited by G Eysenbach; submitted 18.01.17; peer-reviewed by L Mayberry, J Fleming, T Penders, P Musiat; comments to author 22.03.17; revised version received 22.05.17; accepted 24.05.17; published 03.08.17

Please cite as:

Proudfoot J, Clarke J, Gunn J, Fletcher S, Sanatkar S, Wilhelm K, Campbell L, Zwar N, Harris M, Lapsley H, Hadzi-Pavlovic D, Christensen $H$

A Web-Based Public Health Intervention to Reduce Functional Impairment and Depressive Symptoms in Adults With Type 2 Diabetes

(The SpringboarD Trial): Randomized Controlled Trial Protocol

JMIR Res Protoc 2017;6(8):e145

URL: http://www.researchprotocols.org/2017/8/e145/

doi: $10.2196 /$ resprot.7348

PMID: $\underline{28778848}$

CJudy Proudfoot, Janine Clarke, Jane Gunn, Susan Fletcher, Samineh Sanatkar, Kay Wilhelm, Lesley Campbell, Nicholas Zwar, Mark Harris, Helen Lapsley, Dusan Hadzi-Pavlovic, Helen Christensen. Originally published in JMIR Research Protocols (http://www.researchprotocols.org), 03.08.2017. This is an open-access article distributed under the terms of the Creative Commons Attribution License (https://creativecommons.org/licenses/by/4.0/), which permits unrestricted use, distribution, and reproduction in any medium, provided the original work, first published in JMIR Research Protocols, is properly cited. The complete bibliographic information, a link to the original publication on http://www.researchprotocols.org, as well as this copyright and license information must be included. 